\title{
Consecuencias de la legislación en el consumo alimentario de lagartos. El caso del fardacho ${ }^{1}$
}

LUIS CANTARERO

Equipo de Investigación Multidisciplinar en Alimentación Humana Universidad de Zaragoza

\section{RESUMEN}

En este artículo se analiza el caso del fardacho, una variedad de lagarto que se cazaba y consumía en la zona de Peñalba (Huesca). En primer lugar, se describe la cultura alimentaria del fardacho y las razones que motivaron un descenso en el número de ejemplares de este animal. En segundo lugar, se analizan las consecuencias de la legislación sobre especies protegidas en el consumo de fardachos en Peñalba.

\section{SUMMARY}

The fardacho is a variety of lizard that people in the area of Penalba (province of Huesca, Spain) used to hunt and eat. The author describes the food culture to which the eating of fardacho belonged and the reasons that caused a decrease in the number of these animals in the area. In addition, the author discusses the effects of wildlife-protecting legislation on the eating of this lizard in Peñalba.

Palabras clave: Lagarto. Fardacho. Legislación. Cultura alimentaria. Consumo.

Key words: Lizard. Fardacho. Legislation. Food culture. Consumption.

\section{INTRODUCCIÓN}

El ingreso de España en la Comunidad Económica Europea (1986) trae consigo la inclusión en su ordenamiento jurídico de las directivas europeas sobre protección de la fauna y la flora. Este hecho, junto con el movimiento

\footnotetext{
${ }^{1}$ Quiero agradecer a Luis Lax, Carlos Carreras, Begona Royo, David Pac y a los pastores y agricultores de Peñalba su colaboración como informantes. A Marcos Castillo le agradezco una vez más la corrección del texto y sus sugerencias.
}

RDTP, LVI, 2 (2001): 99-112 
social a favor de la conservación de la naturaleza, tiene como respuesta política la promulgación de la Ley 4/1989 de 27 de marzo, de Conservación de los Espacios Naturales y de la Flora y Fauna Silvestres, que trata de frenar el agotamiento de los recursos naturales, la desaparición de especies de flora y fauna y la degradación de los espacios naturales.

Esta ley, en su título IV, dice: "queda prohibido dar muerte, dañar, molestar o inquietar intencionadamente a los animales silvestres [...], incluyendo su captura en vivo y la recolección de sus huevos o crías [...]”.

Estos animales silvestres a los que se aplica el título IV de la ley quedan recogidos en el Catálogo Nacional de Especies Amenazadas. El Estado permite asimismo a las Comunidades Autónomas establecer también, para sus ámbitos territoriales, catálogos similares. Aunque el fardacho $^{2}$, que es el asunto que aquí nos interesa, no se incluye ni en el Catálogo Nacional, ni en el Autonómico de Especies Amenazadas su pertenencia a la categoría de "especie silvestre" hace que este animal esté protegido por la citada ley.

En la Comunidad Autónoma de Aragón, esta legislación afecta a los habitantes de las áreas donde, tradicionalmente, este reptil ha sido utilizado como alimento humano. Entre ellas, figura la localidad de Peñalba ${ }^{3}$. Por este motivo, en este artículo hemos analizado el papel que ha jugado el fardacho en la cultura alimentaria de sus habitantes, las razones que provocaron el descenso en el número de ejemplares de este animal en el entorno de esta localidad y los factores que han motivado que, en la actualidad, haya perdido su función alimentaria humana. En cuanto a este último objetivo, hemos partido de la hipótesis de que, a pesar de lo que se pueda creer, no es la regulación legislativa citada anteriormente lo que ha provocado la desaparición del consumo de fardachos en Peñalba, sino un cambio en la cultura alimentaria de sus habitantes.

\footnotetext{
${ }^{2}$ El nombre científico del fardacho es lacerta lepida. Su nombre vulgar es lagarto ocelado. Popularmente se le denomina también gardacho o fardacho (según las zonas geográficas). En este artículo hemos adoptado esta última denominación, debido a que en Peñalba, lugar donde hemos recogido los datos - véase nota 3-, lo conocen por este nombre. Según J. Corominas y J. A. Pascual (1980), el nombre de fardacho proviene del cruce del árabe hardûn 'lagarto' con el preislámico valenciano de origen bizantino sarvacho. El primer documento donde aparece el fardacho como palabra provincial es de 1817, aunque un derivado suyo, fardachina ('lagartija'), ya figura dos veces en un inventario aragonés de 1374.

3 Peñalba está situada en la provincia de Huesca, en la comarca de los Monegros. Actualmente, según el Padrón Municipal del año 1996, cuenta con 817 habitantes de población de derecho, de los cuales 409 son mujeres y 408 son hombres. Su principal fuente de ingresos proviene de la agricultura y la ganadería, y de las empresas de transporte.
} 


\section{EL FARDACHO COMO ALIMENTO HUMANO}

El fardacho se encuentra en la práctica totalidad de la Península Ibérica, en la mitad sur de Francia, en el noroeste de Italia y en el norte de África (Arnold y Burton 1978; Mateo 1997; Salvador 1998). En Aragón, aunque se pueden encontrar en los valles altopirenáicos, es más abundante en las zonas bajas.

Este animal habita en territorios con grandes precipitaciones y también en regiones áridas. Se refugia entre las matas y arbustos, cerca de cultivos y caminos, en áreas rocosas o pedregosas, bordes de carreteras, madrigueras de conejos o en otros escondites que él mismo fabrica.

El fardacho ocupa un lugar importante en el equilibrio ecológico como presa habitual de numerosos carnívoros y como predador. Su color principalmente es verde con manchas azules, denominadas ocelos, en el costado. Aunque puede alcanzar hasta $80 \mathrm{~cm}$., en general su longitud es mucho menor. En libertad puede vivir más de once años, mientras que en cautividad alcanza los diecisiete. La época del celo comienza en abril y se prolonga hasta junio. La hembra, dependiendo de su tamaño, pone un número variable de huevos, entre 5 y 22 , y las crías nacen durante el verano.

Es un lagarto diurno, y su actividad se desarrolla en las horas centrales del día hasta la puesta del sol. Aunque la mayor parte de esta actividad se desarrolla en el suelo, trepa bien a las rocas y a los árboles. Se alimenta principalmente de pequeños y grandes insectos, lagartijas, gusanos, crías de pájaro y conejo, y fruta ${ }^{4}$.

Una de las características de este animal, que raras veces se describe en los trabajos que hablan de él, es su función como alimento humano. Los habitantes de Peñalba son algunos de los que, durante años, supieron sacar partido de esto. Los más ancianos del lugar aseguran que lo han comido "desde siempre" y que dejaron de hacerlo a finales de los años 70 y principios de los $80^{5}$.

\footnotetext{
${ }^{4}$ Acerca de las características enumeradas anteriormente sobre la lacerta lepida (fardacho), véase Salvador (1998) y Mateo (1997).

5 Sobre la historia del consumo de lagarto, en general, hemos encontrado alguna referencia en Corbier (1999) y Rosenberger (1999). Según el primero, entre los restos arqueológicos romanos hallados en el desierto egipcio, no lejos del mar Rojo, se han encontrado lagartos. El segundo deja constancia de que este animal también fue consumido por los pastores nómadas de la época preislámica. Gálvez, Morales y Castañeda (1999) señalan que el consumo de lagartos era también propio de los indígenas americanos.
} 
La caza fue el medio utilizado por los habitantes de Peñalba para conseguirlo. Sin embargo, no era una caza regulada por la Administración, sino, como dicen en este pueblo, era una captura "oportunista", "accidental" y "no programada". Es decir, lo cazaban los agricultores y pastores cuando, trabajando en las labores del campo, se lo encontraban por casualidad. Debido a que tradicionalmente estos oficios han sido desempeñados por los hombres, la caza del fardacho estaba asociada al género masculino, aunque si algunas mujeres, faenando por el monte, se encontraban con uno también hacían lo posible por capturarlo. No obstante, la caza no siempre era tan casual como puede parecer de lo dicho anteriormente. Los hombres reconocen que a veces suspendían su actividad laboral para "ir a por éln: removían intencionadamente piedras, arbustos, matorrales, etc., para encontrar alguno de estos reptiles. Además algunos pastores disponían de "perros fardacheros", denominados así porque tenían una especial capacidad para localizarlos. Habitualmente, la caza acontecía cuando el fardacho sale de su estado de ivernación (dependiendo del frío, ivernan entre octubre y febrero), pues en ese momento se encuentran "atontados" y por eso es más fácil capturarlos. Se cazaban de día y nunca de noche, ya que, como dijimos anteriormente, su actividad decae con la puesta del sol.

Una vez localizado en el monte, las formas de cogerlo eran variadas. Según aseguran los pastores de esta zona, nunca se cazaba con escopeta. Algunas veces se utilizaban cepos que se colocaban, según un informante, alli donde había abundantes hormigas, ya que al fardacho le gustan estos animales. Lo más habitual, sin embargo, era correr tras él hasta arrinconarlo (en ocasiones no había necesidad de ello, ya que durante la persecución se le tiraban piedras y los más afortunados lograban malherir e incluso matar al animal). Una vez acorralado, se le ponía el mango de la navaja o un palo en la boca (los más valientes les metían el dedo gordo de la mano). El fardacho lo mordía instintivamente y no lo soltaba durante un tiempo suficiente para darle un golpe en la cabeza y provocar su muerte.

Una vez capturado, jamás se comercializaba. Lo normal era consumirlo en el monte el mismo día de la captura. En ocasiones se llevaba al pueblo para compartirlo con la familia y otras veces se regalaba a amigos o a "notables" del pueblo con el propósito de devolver favores o asegurárselos en el futuro.

En el monte, el fardacho era cocinado y consumido por los hombres, ya que eran ellos los que normalmente faenaban en el campo y los que lo capturaban. Cuando un pastor o un agricultor cazaba uno, lo habitual era compartirlo con sus compañeros de trabajo ${ }^{6}$. Esta situación propor-

\footnotetext{
${ }^{6}$ Los comidas colectivas en el monte eran habituales entre la gente que laboraba en el campo. Aquí pasaban largas temporadas sin volver a casa. Los pastores y los
} 
cionaba placer a los comensales, ya que el consumo "Ocasional" de este reptil satisfacía el deseo de variedad de estos sujetos, acostumbrados a una alimentación rutinaria, funcionaba como cohesionador del grupo, pues tenía la facultad de reunir a los pastores y agricultores, y reforzaba su orgullo de pertenencia gremial y rural. Además, su forma de prepararlo, a la brasa, asado en el fuego, sugería a los comensales, varones todos ellos, nociones culturales llenas de significado. Como dice Montanari (1993: 35), “[...] los antropólogos nos han enseñado que la imagen de la comida asada en el fuego, directamente a la llama, corresponde a unas nociones culturales muy distintas de las que sugiere el agua hirviendo en la olla: nociones de violencia, impetuosidad y belicosidad, de una compenetración más 'salvaje' con la naturaleza. También González Turmo (1995: 221), señala que "pesa del lado del varón la relación alimenticia con el campo, con la naturaleza, y, desde luego, con el fuego".

Una vez destripado, se colocaba en la parrilla. Cualquier pastor lo cocinaba y no había ninguno que estuviera especialmente dotado para llevar a cabo este menester. Durante este proceso, los pastores se aseguraban también de extraer los riñones del animal, antes de cocinarlo, con la punta de la navaja, ya que aseguraban que ingerirlos "era malo para la salud". Según creían, quienes los consumían tenían problemas para orinar.

El fardacho se cocinaba entero, aunque muchas veces sin la cola, ya que el animal la desprende para engañar a sus depredadores. Curiosamente, ésta era una de las partes del fardacho más apreciada, debido a que "no tenía casi hueso y era muy tierna". Otra de las piezas más valoradas eran las cocochas del cuello. Por ello, una vez degollado el animal, no se tiraba su cabeza. El fardacho se colocaba en el fuego sin despellejarlo, "si no, se quema la carne", y una vez asado, antes de comerlo, se le quitaba la piel (en caso de que quedara algún resto, durante la ingestión se escupe de la boca). A la carne que queda al descubierto, se le añadía sal y aceite. Por lo que dicen quienes lo consumían de esta manera, no hacía falta condimentarlo más, ya que el fardacho, por sí solo, es muy sabroso. Una vez preparado, se tomaba para almorzar con un par de huevos fritos o para comer como aperitivo antes del plato principal, generalmente una calderada (carne de caza o cordero con patatas).

En otras ocasiones, el fardacho no se consumía en el monte, sino que se llevaba a casa para ser compartido con la familia o con los amigos. Ahora bien, no lo llevaba a casa el pastor que lo cazaba, sino al que le

agricultores se llevaban grandes cantidades de legumbres, tocino, etc., y completaban su alimentación con cualquier animal que pudiera ser cazado. Como decimos, diariamente se reunían para compartir estos alimentos. 
tocaba. Es decir, como si de un turno rotativo se tratara, los pastores compartían este "regalo", eliminando con ello la competencia por la caza y las envidias que pudieran derivar del éxito en la misma.

En casa también se consumía como aperitivo y el mismo día de la captura, o a más tardar al día siguiente, y jamás se conservaba. Sin embargo, una vez que el fardacho entraba en el espacio doméstico, las mujeres decidían su forma de elaboración. Así, lo despellejaban, lo destripaban, lo troceaban y lo freían en la sartén con aceite y ajo ${ }^{7}$. Para un pastor de la zona, el que las mujeres se quejaran de lo costoso de su preparación (según dicen éstas, despellejarlo y destriparlo era muy fatigoso) fue uno de los motivos de lo esporádico de esta práctica alimentaria en el espacio doméstico.

Las diferencias de género se manifestaban no sólo en la forma de preparación, sino también en la valoración general del fardacho. Mientras que para los varones era un plato muy apetitoso, para las mujeres no era tan sabroso (aunque hay algunas que aseguran que a ellas también les gustaba muchísimo). Y esto es así porque como dice González Turmo (1995: 221) la importancia de la carne en las comidas de hombres es indiscutible: "[...] ha sido alimento casi indispensable en sus comidas extracotidianas. Su presencia debía asegurarse a cualquier precio. Y cuando hablo de precio, no hago sólo mención a su valor económico, sino a su rareza o a la superación que supone la ingestión de carnes poco comunes y rechazadas por el resto. Es el caso de lagartos, serpientes, galápagos, burros e incluso gatos, carnes todas ellas que los hombres han comido entre sí [...] para horror y repugnancia de las mujeres, que, por regla general, manifiestan auténtica aversión hacia la posibilidad de ingerir estos animales".

De hecho, el consumo de fardacho en la zona de Peñalba formaba parte de la cultura que los habitantes de esta localidad denominan del "Comer de todo". Los pastores aseguran que en el monte cualquier animal

\footnotetext{
7 Sobre las formas de cazar al fardacho y consumirlo hay obviamente diferencias culturales. En un recetario publicado por la Caja de Ahorros de Navarra (Sarobe 1995), dicho sea de paso, denunciado por enumerar recetas "insólitas" de animales protegidos, se dice que el gardacho (denominado así en Navarra), bien apreciado por gentes de toda condición social y económica, se mata con la escopeta si se sube a un árbol. Si se captura a mano, se lanza al aire y cuando cae se le da un navajazo en la parte posterior de la cabeza. Se despelleja y desviscera para asarlo a la parrilla y se le añade sal y aceite. Hay quien frota su carne con ajo antes de asarla. Se elabora también troceado y frito en aceite con ajo. En algunos lugares de Navarra, el gardacho, una vez troceado, se añade a las judías blancas, o se guisa con tomate o patatas y en fritada de tomate con pimientos.
} 
"era bueno para comer". Entre los animales "raros" que ingerían destacan los erizos, las ratas de campo y, en especial, una gran variedad de aves: esparbel (cernícalo primilla), mochuelos, poputes o abubillas, gangas, ortegas, alcaravanes y toda clase de pájaros. Como dice un pastor, ave que vuela a la cazuelan. Todos estos "bichos raros" que cazaban, junto con legumbres, tocino, vino, etc., formaban parte de la alimentación de pastores y agricultores durante sus largas permanencias en el monte.

\section{ACERCA DEL DESCENSO EN EL NÚMERO DE EJEMPLARES DE FARDACHO}

El descenso en el número de ejemplares de fardacho en la zona de Peñalba no es debido a las prácticas alimentarias de los habitantes, sino a la modernización de las labores agrícolas a partir de la década de los años 60 y a la política agraria de los 80 .

En la década de los 60 se terminaron de mecanizar las labores del campo; las trilladoras, las empacadoras, las cosechadoras, etc., formaban ya parte del paisaje. Uno de los cambios más importantes, debidos a esta progresiva mecanización fue la sustitución de la mula por el tractor ${ }^{8}$. Como consecuencia de este proceso de mecanización, rápidamente se modificó el paisaje: se agrandaron los campos de labor, se juntaron parcelas, se eliminaron piedras del terreno (antes de la mecanización la labranza no implicaba esta práctica), etc., y todo ello repercutió en la pérdida de escondites para el fardacho. A esto hay que añadir que a principios de los años 70 también se extendió el uso de insecticidas, con el consiguiente peligro de envenenamiento para ellos y para los insectos, su principal fuente de alimentación.

Más recientemente, en la década de los 80 , otros factores que alteraron el monte y disminuyeron la superficie de rastrojos, provocando por consiguiente un descenso en el número de ejemplares, fueron la concentración parcelaria, la transformación de las tierras de secano en regadío y la repoblación de las laderas. Durante esta década y la siguiente la administración autónoma decide la concentración de parcelas disminuyendo su número y aumentando su tamaño. Independientemente de sus consecuencias económicas, esta concentración tiene como resultado la destrucción de ribazos, márgenes de campos, etc. Además, como señala J. Bada

\footnotetext{
${ }^{8}$ Los primeros tractores de rueda metálica o de hierro aparecieron en la zona de Peñalba en la década de los años 40. Posteriormente, a principios de los 50, aparecieron los primeros de ruedas de goma. Sin embargo, es en los años 60 cuando se extiende la gran mecanización.
} 
(1999: 205), "a la intervención pública sobre todo el territorio sobrevino en cada parcela adjudicada la intervención privada de los propietarios. La racionalidad de la economía se impuso sobre cualquier otra en ambas intervenciones. Los propietarios particulares se afanaron allanando y subsolando los campos y sacaron muchas piedras que dejaron al margen en grandes montones como siempre habían hecho. Se roturaron agüeras y regueros, se colmataron balsas y balsetas fraguadas, se destruyeron ribazos y saltadores, se arrasaron viejas casejas".

A todos estos factores de orden material hay que añadir otros del orden de las representaciones y las creencias. En la tradición oral de la zona de Peñalba, la relación entre los reptiles y los humanos ha sido de amorodio ${ }^{9}$. En cuanto al fardacho en particular, fue adorado por su función alimentaria y odiado por otros motivos. Más concretamente, se le ha acusado de comerse los huevos de las perdices y, en esta zona, todo lo que pueda hacer descender el número de estos animales ha de ser eliminado. Los guardas de los cotos (empleados municipales) asumían esa responsabilidad: "hacían limpia", "los liquidaban".

Como resultado de todos estos factores materiales y no materiales, descendió el número de fardachos en la zona de Peñalba. No obstante, hoy en día, ni para los lugareños ni para la administración autonómica este animal está amenazado de peligro de extinción. Ambos están de acuerdo en que hay desapariciones y disminuciones locales, pero su presencia es todavía habitual en casi toda la Península Ibérica. Además, en la zona de Peñalba, se confía en volver a ver fardachos corriendo por el monte, gracias a su alto grado de adaptación y a que el campo se ha estabilizado. De hecho, algunos aseguran que ya hoy en día se les vuelve a ver por los alrededores.

\section{LA LEGISLACIÓN Y EL CONSUMO DE FARDACHO}

Acerca de la protección por ley del fardacho cabe preguntarse cómo la interpretan los afectados y en qué medida ha influido en su consumo. En cuanto a la primera cuestión, en Peñalba desconocen la ley que regu-

\footnotetext{
${ }^{9}$ De entre los reptiles, las serpientes son las más odiadas. Este sentimiento se refleja en las historias transmitidas oralmente de generación en generación. Así, por ejemplo, cuentan que en la zona de Peñalba, en una ocasión, una madre amamantaba a su hijo, pero, curiosamente, éste no engordaba. Según pudieron descubrir, era debido a que una serpiente hipnotizaba a la señora y "destetaba" a su hijo ocupando ella su lugar y consumiendo la leche materna. Para que el niño no llorara, la serpiente introducía su cola en la boca del niño. Una vez descubierto el entuerto la mataron.
} 
la su protección, es decir, no saben qué ley la recoge, ni desde cuándo está en vigor. Es a través de los medios de comunicación de masas como se enteran de que no pueden cazarlo, ni consumirlo. Algún habitante escucha una noticia ejemplar y el resto de sus conciudadanos, a través del boca a boca, quedan enterados ${ }^{10}$.

Sin embargo, para los habitantes de esta zona, conocer el origen de la prohibición carece de importancia. Les basta con saber que hay una legislación que prohibe su caza y su consumo, por un supuesto peligro de extinción, para manifestar abiertamente su desacuerdo hacia esta regulación legislativa. Así, sostienen que la ley no debería afectar a los habitantes del medio rural que han convivido tradicionalmente con el fardacho. Es decir, no aprueban que se pueda penalizar el consumo de este reptil (que, como vimos, era esporádico) por parte de un habitante del medio rural, ya que aseguran que esta práctica alimentaria no es la responsable de su supuesta extinción, ni de la flora o la fauna en general. En este sentido se sienten claramente perjudicados y piensan que se les quiere hacer responsables de la destrucción del medio ambiente cuando en realidad no es así. Además, aunque, como veremos más adelante, hoy en día no se consumen fardachos, lamentan que la legislación les impida llevar a cabo o recuperar esta práctica alimentaria en caso de que quisieran.

Añaden que la legislación podría penalizar la caza, pero no su consumo. Con un símil, un pastor de la zona lo resume de la siguiente manera: "Si un niño se compra una pistola hay que castigar al de la tienda, no al niño. Es decir, una vez muerto por qué tirarlo. Como muestra de rebeldía, gran parte de los pastores de Peñalba, que rondan los 60-65 años, y otros curiosos, afirman que en caso de tener ocasión no dudarían en volver a comerlo.

Lo que sí desaprueban es el carácter mercantilista que podría tomar su consumo en caso de que no estuviera protegido. Así, apoyan que la comercialización sea ilegal, ya que "si no estuviera prohibido, habría algún restaurante en la Diagonal ${ }^{11}$ que lo vendería y ganaría mucho dinero". En definitiva, agradecen a la ley el ser un mecanismo eficaz contra su uso comercial, ya que según su mentalidad este animal no puede ser mercantilizado.

${ }^{10}$ Como todos los rumores, el de la prohibición del consumo de fardacho probablemente también se ha distorsionado. En Peñalba, por ejemplo, se corre la voz de que en Madrid le impusieron a uno una multa de 500.000 pts. por cazar un fardacho. Otros, sin embargo, aseguran que la multa fue de 1.000.000 de pts. En cualquier caso, se da por hecho que cazar un fardacho puede costar muy caro.

${ }^{11}$ Avenida famosa de la ciudad de Barcelona. 
En cuanto a la segunda de las preguntas planteadas, que el terror a la sanción económica ${ }^{12}$ reprime actualmente la caza del fardacho para consumo alimentario humano parece evidente. Sin embargo, hay otras razones más importantes que han motivado la desaparición de este animal de las prácticas alimentarias de los habitantes de Peñalba.

Al igual que es difícil definir con precisión el origen de su consumo, lo es delimitar el abandono de esta práctica. Los habitantes de Peñalba señalan que hacia finales de los años 70 ó principios de los 80 dejaron de verse fardachos por el monte, debido a los factores enumerados anteriormente, y, por lo tanto, eliminaron de sus hábitos alimentarios su ingesta.

En cualquier caso, cuando la ley viene publicada en el Boletín Oficial del Estado nos hallamos en 1989 y para esta fecha el fardacho es un símbolo de un tiempo ya pasado. Así, se puede asegurar que el efecto de la ley sobre este consumo en el medio rural no tiene ninguna consecuencia, una vez que la cultura alimentaria de los habitantes de Peñalba ha modificado el estatus de este animal.

Como dijimos, la modificación del habitat, como consecuencia del uso generalizado de la maquinaria, comenzó a poner en peligro su supervivencia. Sin embargo, este peligro no fue razón suficiente para explicar el hecho de que, durante este período, disminuyera el interés por su caza y, por lo tanto, por su consumo. Esta pérdida de interés estuvo relacionada con los cambios producidos en el estilo de vida de los habitantes de Peñalba como consecuencia de la propia evolución temporal de la cultura y de la mecanización. Así, por ejemplo, una de las razones que motivó una menor dedicación a la caza del fardacho es que ir andando al monte y capturarlo eran prácticas asociadas, lo que ya no tenía sentido con la introducción del tractor.

Además, el uso generalizado de las máquinas permite abarcar mayor extensión y ganar terreno productivo, lo que trae consigo una mayor dedicación al trabajo y una pérdida de motivación hacia su captura. Como asegura un informante, "te dedicas a hacer mil faenas y no tienes tiempo para entretenerte a cazarlo [...] es una cuestión de productividad". Durante esta época, cambia el concepto de rentabilidad. Se introducen ulas prisas" en el campo y desaparecen las comidas colectivas. Los buenos resultados económicos de los años 60, consecuencia de esta mecanización, fueron también resultado de las abundantes lluvias que cayeron en la zona de Peñalba. Como consecuencia, se invirtió más tiempo en cultivar más tierra y menos en cazar el fardacho.

${ }^{12}$ El título VI de la ley 4/1989 de 27 de marzo de Conservación de los Espacios Naturales y de la Flora y Fauna Silvestres recoge las infracciones y sanciones para quienes la infrinjan. Las sanciones económicas van desde 10.000 hasta 50.000 .000 ptas. 
A todo esto hay que añadir que las máquinas mediatizan la relación con la naturaleza. Es decir, que la relación entre el hombre y la tierra ya no es tan cercana, ni física ni simbólicamente, como lo era antes de la mecanización, por lo que el papel del fardacho, asado al fuego, como sinónimo de nociones culturales de violencia, impetuosidad, etc. (Montanari 1993), pierde importancia.

Todas las innovaciones materiales que afectaron al trabajo de la tierra vinieron acompañadas por cambios en la organización social rural, en la forma de vida y en los valores socioculturales. Desde mediados de los sesenta, en la zona de los Monegros, va modificándose la organización de la familia, el sistema de transmisión hereditaria, la administración de la hacienda, el modo de relacionarse los parientes entre sí o con los vecinos, etc. (Bada 1999: 61). A todo esto acompaña el acceso de la mujer a la educación y a puestos de trabajo en la ciudad, el desprestigio de lo rural, el atractivo por la vida urbana, la pérdida del sector agrario en términos económicos, la movilidad social, los modernos ideales de autonomía, el deseo de mayor libertad, etc. (Bada 1999: 69).

Qué duda cabe que todo ello afecta a la cultura alimentaria en general y a las representaciones en torno a la carne en particular. Es decir, como dice Millán (1998: 139), "sociedad y cultura varían en el tiempo y en el espacio: por tanto también se modifica la imagen del animal y su interacción con los humanos".

En el caso del fardacho se ha producido un desplazamiento en su estatus alimentario y un cambio en su significado simbólico. Es decir, se han modificado las representaciones en torno al objeto-animal fardacho. Si en otros tiempos era altamente valorado como alimento humano, hoy en día las razones que motivaban este aprecio han desaparecido y, por lo tanto, ha decrecido el interés por su captura. Y es que, cómo se van a molestar en ir a por un fardacho si las comodidades que garantiza la mecanización hacen que el agricultor prefiera comerse un bocadillo escuchando la radio y con aire acondicionado antes que ir en su busca. Además, tanto el agricultor como el pastor comen habitualmente en casa, por lo que las comidas colectivas en el campo, en las cuales el fardacho ocupaba un lugar capital, pierden importancia. Este hecho, junto con que no se vieran tantos por el monte y con la bonanza económica de los años 60, han sido las razones fundamentales, para los habitantes de Peñalba, del desuso del consumo de estos reptiles.

Sin embargo, como adelantamos anteriormente, las personas que rondan los 60-65 años y participaron de la "cultura del comer de todo", y otras más jóvenes fuertemente relacionadas con la cultura rural, aunque no demuestren un gran interés por su captura, aseguran que, como cosa 
"pintoresca", si se tercia lo volverían a consumir ${ }^{13}$. Se consumiría, por supuesto, a escondidas, entre los amigos, no en público, y jamás se pronunciaría el nombre del responsable de su captura.

Quienes no lo consumirían jamás serían las nuevas generaciones. A los miembros de la "cultura del comer de todo", les han sucedido los de lo «bueno para la salud. Despectivamente se dice que ahora al campo se llevan yogures, salmón ahumando y coca-colas. Para estos consumidores modernos, la ingestión de fardacho ha caído en tan fuerte descrédito que provoca repugnancia. De hecho a los jóvenes les repele la sola idea de consumirlo. Como vemos, el tiempo ha modificado lo culturalmente apetitoso. Este sentimiento, que es moral y social (Miller 1998: 22), proviene, entre otras razones, del proceso de humanización del animal ${ }^{14}$, que es uno de los factores que ha contribuido notablemente a modificar la posición subjetiva de los humanos con respecto a los animales. Según Millán (1998: 148), "hoy asistimos a un proceso de movilidad socioanimal ascendente (humanización). En el ámbito de los países postindustriales, dicho proceso lo podemos observar en la actualidad. Se subrayan las cualidades del animal, se proclaman sus derechos, asociaciones voluntarias los defienden, aumentan las especies protegidas, se intenta encubrir los procesos anteriores al consumo, etcétera".

Por ello, los jóvenes no se molestan en capturar un fardacho, ya que "la gente está mejor comida", "aunque tiene cancha, es carne de segunda categoría", y no hay necesidad de consumir animales "raros", característicos de una época ya pasada. Si "antes valía cualquier cosa para comer" hoy hay donde elegir. Y, puestos a hacerlo, se prefiere comer la carne que proviene de la caza habitual (liebre, conejo y ciervo) o la de venta en las carnicerías (ternera, cerdo, etc.). La de fardacho marca una frontera generacional: la de los ancianos y su "comer de todo", con la de los jóvenes y la modernidad.

\section{CONCLUSIÓN}

El fardacho, y su estatus alimentario en el pasado, es un tema recurrente en Peñalba. Surge a menudo en las charlas de café y siempre se habla de él de una manera positiva. Se ensalza su sabor y trae a la

${ }_{13}$ Uno de estos sujetos recuerda que la última vez que comió fardacho fue a principios de los 90, es decir pocos años después del establecimiento de la legislación proteccionista.

${ }^{14}$ Acerca de este proceso de humanización del animal, véase Millán (1998). 
memoria infinidad de situaciones placenteras vividas por los comensales, habitualmente hombres, que participaban en su consumo.

Su prohibición por ley ha influido en que esto sea así. De ser una práctica alimentaria abandonada mucho antes de la entrada en vigor de esta ley, debido, como hemos visto, a los cambios sociales y culturales habidos en la zona de Peñalba a partir de los años 60 , y recordada en ocasiones esporádicas, ha pasado a ser, como decimos, un tema de actualidad.

Se discute acerca del porqué de la legislación y los habitantes de Peñalba expresan opiniones a favor y en contra de la misma. Por un lado, se lamentan de no poder recuperar un hábito alimentario en caso de que quisiesen $y$, por otro, agradecen que tampoco puedan hacerlo los sujetos que no pertenecen a su medio rural y que tengan fines comerciales.

Todo esto apunta a que la legislación protege la flora y la fauna e incide en la cultura alimentaria. Estamos en una época en que el proceso de humanización del animal (Millán 1998) hace que el poder legislativo priorice la protección de las especies animales y vegetales sobre las prácticas alimentarias humanas. La pregunta que se plantea es cómo se podría compaginar la protección de especies silvestres y de prácticas culturales humanas, máxime cuando, actualmente, en el caso del fardacho, la razón que supuestamente justificó la legislación -a saber: el riesgo de peligro de extinción - no tiene sentido. Sin embargo, aunque la legislación dejara una puerta abierta a la puesta en práctica de nuevo de su consumo, las modernas tendencias en alimentación la cerrarían. No obstante, quién sabe si en el futuro, en los jóvenes habitantes de Peñalba, resurgirá de nuevo el interés por recuperarlo, máxime cuando los valores de la tradición, de la naturaleza, etc., vuelven a estar de actualidad en alimentación y hay una tendencia a recuperar platos hace un tiempo abandonados.

\section{BIBLIOGRAFÍA CITADA}

ARNOLD, E. N. y J. A. BURTON. 1978. Guia de campo de los reptiles y anfibios de España y de Europa. Barcelona: Omega.

BADA, José. 1999. Una cultura del agua en los monegros. Zaragoza: Egido.

CORBIER, M. 1999. "La fève et la murène: hiérarchies sociales des nourritures à Rome", en J. L. Flandrin y M. Montanari (dirs.), Histoire de l'alimentation: 215-236. Paris: Fayard.

COROMINAS, JOAN y J. A. PASCUAL. 1980. Diccionario crítico-etimológico castellano e bispánico. Madrid: Gredos.

Gálvez, C. A., R. MORAles y J. Castañeda. 1999. "11.000 años de consumo de reptiles en la costa norte del Perú: el caso del Cañán (Dicrodon sp.)n, en A. Garrido Aranda (comp.), Los sabores de España y América: 141-164. Huesca: La Val de Onsera. 
González Turmo, ISABEl. 1995. Comida de rico, comida de pobre. Sevilla: Universidad de Sevilla.

MAteo, J. A. 1997. "Lacerta lepida", en J. M. Pleguezuelos (ed.), Distribución y biogeografía de los anfibios y reptiles en España y Portugal: 222-224. Granada. Universidad de Granada.

Millán, AMADO. 1998. "Acerca del status animal". Estudios del Hombre 7: 133-149.

Miller, William IAN. 1998. Anatomía del asco. Madrid: Taurus.

MONTANARI, MASSIMO. 1993. El bambre y la abundancia. Historia y cultura de la alimentación en Europa. Barcelona: Crítica.

ROSENBERGER, B. 1999. "La cuisine arabe et son apport à la cuisine européenne", en J. L. Flandrin y M. Montanari (dirs.), Histoire de l'alimentation : 345-365. Paris: Fayard. SAlVADOR, AlFREDo (coord.). 1998. Fauna Ibérica. Reptiles. Vol. 10. Madrid: Museo Natural de Ciencias Naturales. CSIC.

SAROBE, VÍCTOR MANUEL. 1995. La cocina popular navarra. Pamplona: Caja de Ahorros de Navarra. 